\title{
Linx
}

Revue des linguistes de l'université Paris X Nanterre

56 | 2007

Linguistique des genres

\section{Les discours de la presse quotidienne : observer, analyser, comprendre, Sophie Moirand}

Presses Universitaires de France (PUF), 2007, Paris, 179 p.

\section{Gérard Petit}

\section{(2) OpenEdition}

\section{Journals}

Édition électronique

URL : http://journals.openedition.org/linx/386

DOI : $10.4000 /$ linx.386

ISSN : 2118-9692

\section{Éditeur}

Presses universitaires de Paris Nanterre

Édition imprimée

Date de publication : 1 juin 2007

Pagination : 197-202

ISSN : 0246-8743

\section{Référence électronique}

Gérard Petit, "Les discours de la presse quotidienne : observer, analyser, comprendre, Sophie Moirand", Linx [En ligne], 56 | 2007, mis en ligne le 22 février 2011, consulté le 22 septembre 2020. URL : http:// journals.openedition.org/linx/386 ; DOI : https://doi.org/10.4000/linx.386 


\section{Compte rendu}

Gérard PETIT, Université de Paris X. Les discours de la presse quotidienne : observer, analyser, comprendre, Sophie Moirand., Presses Universitaires de France (PUF), 2007, Paris, $179 \mathrm{p}$.

La lecture de cet ouvrage ne peut qu'être profitable pour qui s'intéresse à l'Analyse du discours, à la Linguistique textuelle mais aussi à la Lexicologie. En effet Sophie Moirand a fondé et dirigé pendant de nombreuses années le CEDISCOR (Centre d'Etude sur les Discours Ordinaires et spécialisés) à l'Université Paris III Sorbonne-Nouvelle. La prééminence de l'auteur dans le paysage de l'Analyse du discours (désormais $\mathrm{AD}$ ), n'est plus à démontrer. Son abondante bibliographie atteste de son activité et de la qualité de sa recherche. Les discours de la presse quotidienne se présente comme un bilan des positions de l'auteur, de ses travaux en $\mathrm{AD}$, une application sur corpus des notions qu'elle a travaillées et développées. Il constitue en même temps une prospective pour une orientation nouvelle de la recherche en AD, qui serait davantage centrée sur les modalités de circulation des dires, leur interpénétration, ainsi que sur la prise en compte des entrées lexicales sur lesquelles se construisent les dires (raison pour laquelle l'AD n'est jamais totalement déconnectée d'une forme de Lexicologie). Enfin, dernier intérêt présenté par l'ouvrage : remédier à une carence de manuels fondés sur une perspective moderne et des recherches récentes.

Le texte se compose de quatre chapitres :

1. Les choix de l'analyse

2. La circulation des mots et des formulations

3. La diversité des rondes et des dires

4. Mémoires et médias

S'y ajoute un très utile index des notions qui, à lui seul, constitue une conceptologie de base opératoire pour toute recherche en analyse du discours. Le 
corpus d'analyse est constitué de textes empruntés à la presse quotidienne, recueillis durant des «moments discursifs » (cf. plus bas).

Le propos respecte une progression qui lui confère une forte dimension méthodologique :

- dans un premier temps, l'auteure aborde les questions afférentes à la sélection et à l'explicitation des options théoriques et méthodologiques nécessaires à l'analyse et précise ses choix (Chap. 1);

- ensuite la description se centre sur le substrat linguistique des discours : les mots, les formulations et les prérequis qui président à leur instanciation (Chap. 2)

- en troisième lieu, la perspective s'élargit pour interroger la circulation des énoncés, leurs interrelations respectives, lesquelles vont contribuer à la diffusion de ces mots et formulations, à leur investissement sémantique et à la concrétisation des discours (Chap. 3)

- enfin elle se porte sur la détermination de deux principes majeurs qui conditionnent la production et la circulation des discours: la mémoire (des locuteurs mais aussi des mots qu'ils emploient) et le dialogisme inhérent à toute mise en verbe (Chap. 4).

Le premier chapitre pose les prémisses théoriques et méthodologiques de la démarche. L'auteure procède en trois temps. D'abord l'exposition d'une notion de base qui est appelée à conditionner l'élaboration du corpus, celle de moment discursif: «un fait ou un événement ne constitue un moment discursif que s'il donne lieu à une abondante production médiatique et qu'il en reste également quelques traces à plus ou moins long terme dans les discours produits ultérieurement à propos d'autres événements » (p. 4). Les échantillons d'analyses reproduits dans l'ouvrage sont empruntés à des productions émises durant des moments discursifs récents qui ont agité le débat politique, économique et médiatique. Pour n'en citer que quelques uns : l'affaire du sang contaminé, la crise de la vache folle, la grippe aviaire, les OGM dans l'alimentation, l'arrachage de plants de maïs transgénique... Ensuite viennent les questions liées à l'élaboration elle-même de ce corpus (quels types de sources, quels matériaux pour l'analyse au sein des productions émanant de ces sources, quelles fragmentations fonctionnelles opérer sur le corpus, pourquoi et comment?). Sur le plan méthodologique, la condition d'une analyse de discours est définie au moment de la constitution et de la définition du corpus de données. On n'insistera jamais assez sur le caractère primordial de cette étape, qui conditionne en sortie la configuration et l'identité même des résultats. C'est l'un des mérites de cet ouvrage de revenir sur ce point et de détailler les contraintes imposées à l'élaboration du corpus d'analyse. C'est ainsi que l'on est conduit à distinguer entre corpus de référence, corpus de travail et corpus exploratoire. Historiquement, les réflexions portant sur la constitution et la détermination des corpus ont été menées initialement en $\mathrm{AD}$ à la fin des années soixante. Elles ont été reprises par les travaux en analyse automatique et documentaire pour l'élaboration de leurs outils et de leurs procédures de traitement. On ne pourra qu'apprécier la place qui est donnée à cette problématique dans l'ouvrage de Sophie Moirand, l'AD ayant eu parfois tendance à la perdre de vue. 
Enfin le chapitre se clôt sur une réflexion sur l'identité des unités discursives circulant à l'intérieur de ce même corpus, dénominations, désignations, configuration lexicosémantique de ces séquences. Est ainsi dégagée une typologie des unités discursives avec une mise en relief de leur hétérogénéité : hétérogénéité sémiotique, hétérogénéité textuelle et hétérogénéité énonciative. Sophie Moirand ne cherche donc pas à proposer ne vision unifiée des données, des observables et des unités qui les composent mais à les exposer dans leur diversité et leur labilité constitutives.

Le second chapitre est consacré à la fonction qu'occupent les mots et formulations dans les discours ainsi qu'à leur nécessaire prise en compte par et pour l'AD. Il aborde en premier lieu les paradigmes désignationnels, notion élaborée initialement par Marie-Françoise Mortureux à la fin des années quatrevingts, que le CESDISCOR a travaillée pour l'appliquer à l'AD dans l'étude des stratégies de reformulation d'une même donnée au sein d'un même discours ou entre discours différents (cf. notamment les travaux de Sandrine Reboul-Touré). Deux autres notions, spécifiques à l'auteure, sont ensuite exposées : celle de motargument et celle de mot-événement. Les mots-arguments correspondent à des notions phares dans l'argumentation développée à l'intérieur de certains discours. La nomination qu'ils instancient acquiert une valeur pragmatique forte : traçabilité, transparence et principe de précaution ont ainsi fonctionné alternativement comme arguments d'autorité et de vente durant la crise de la vache folle afin de rassurer le consommateur, ce qui leur a valu d'être convoqués une nouvelle fois durant celle de la grippe aviaire. Le mot-argument est (re)connu par le récepteur et son emploi est fréquemment l'objet d'allusions ; il est le lieu et l'enjeu d'une connivence entre locuteurs, dispensant ainsi le discours de produire des explicitations, donc d'exposer clairement ses prérequis ainsi que le cadre juridique (au sens large) qui lui permet de se déployer et de circuler. Le mot-événement est une dénomination complexe dont le statut lexical est analogue à celui d'un nom propre : «En prenant comme objets d'étude des moments discursifs médiatiques, on voit surgir en effet des mots et des expressions qui finissent par devenir le 'nom' de ces événements » (p. 56). Le mot événement adopte souvent la forme d'une périphrase ou d'un syntagme réalisés sur le mode de la description définie: le sang contaminé, la vache folle, le prion, la grippe aviaire, le maïs transgénique, les OGM... Ces séquences sont toutes polysémiques et renvoient, dans l'une de leurs acceptions, à une signification paraphasable par «l'affaire du / de la $+\mathrm{GN}$ ». Elles se stabilisent et acquièrent une valeur événementielle, référant non pas à ce que leur structure lexicale laisse induire a priori mais à l'événement même qui s'est tissé autour (le phénomène est proche de ce qui est entendu en sémantique lexicale par nom d'événement). Elles sont chargées des significations qui ont accompagné leur émergence au sein des discours qui les ont produites et qui continuent de les employer.

Le troisième chapitre est doté d'un intitulé volontairement ambigu, à la manière d'un jeu de mots : «la ronde des dires». Par «ronde» il convient d'entendre à la fois "circulation", dans son acception de "transmission" mais aussi, 
dans un sens plus étymologique de "circularité" en ce que les dires se diffusent et se reprennent mutuellement en un incessant brassage. Ce mode de circulation endocentrique est analysé dans le détail. Deux caractéristiques doivent ici retenir l'attention: la composante dialogique des discours et la structuration trilogale de leur conditions de circulation. La structure dialogique des discours a donné lieu à d'abondantes publications en $\mathrm{AD}$ à partir des travaux de Bakhtine. Les analyses influencées par les théories de l'argumentation avaient eu tendance à s'éloigner quelque peu de cette perspective, qui oblige non plus à percevoir le discours comme une unité compacte et autocentrée, mais comme un espace ouvert et traversé par une pluralité de dires, un interdiscours, qu'il brasse et cite de manière plus ou moins explicite, montrée ou cachée. La situation trilogale de transmission des savoirs dans les médias est décrite dans sa fonction pragmatique : quel jeu joue à l'intérieur des discours de transmission des connaissances le troisième pôle d'énonciation, le journaliste, dans la relation qui unit le détenteur du savoir ou de l'autorité et le public (en principe non averti ou du moins ne disposant pas des mêmes des lecteurs? Les conditions de circulation et de publication des dires dans la presse quotidienne (notamment par le fait que les sources se citent, que les lecteurs ne se satisfont plus d'une seule source, par la présence d'internet qui accentue l'interpénétration de ces sources) font que ce troisième pôle a vu sa fonction évoluer depuis le temps où l'on pouvait le considérer comme un simple intermédiaire cantonné dans une activité de reformulation / simplification, c'est-àdire de traduction intralinguale destinée à un public non averti. L'hétérogénéité profonde des lectorats et de leurs attentes conduit la parole médiatique à s'orienter vers un jeu de positionnements pragmatiques au sein duquel la monstration ou la dissimulation des dires (révélés néanmoins sur le mode de l'allusion) constituent l'enjeu fondamental de cette parole. Loin d'être un agent de vectorialisation, le troisième pôle de la situation trilogale contribue à amplifier la ronde des dires quand ce n'est pas tout simplement à permettre sa possibilité.

Le quatrième chapitre explicite la construction du dialogisme en en décrivant les paramètres constitutifs. Il est ainsi possible de dresser une sorte de typologie des différentes formes de dialogisme, en s'appuyant sur des questionnements simples (p. 120-125), à même de les mettre à jour. La seconde partie de ce chapitre est consacré au rôle de la mémoire dans la circulation et l'interprétation des mots. On sait que la mémoire occupe une place très importante dans l'acquisition de la dénomination et dans les processus d'interprétation. L'idée défendue par l'auteure est que les mots se chargent en discours de valeurs sémantiques, qui ne sont pas nécessairement descriptives, qui reflètent l'événement qu'ils décrivent ou dont leur référent est un constituant, mais aussi les conditions de leur production. Leur fonction référentielle est nourrie par l'instant historique qui a vu leur émergence et dont ils portent la trace. Des unités comme far-west (dans son sens figuré), OGM, principe de précaution, charter, odeur, détail etc. voient leur signifié se charger d'une mémoire discursive intersubjective, socialement stabilisée et inscrite dans leur valeur lexicale, de manière parfois analogue à celle d'une étymologie. De fait les mots en discours acquièrent une indexicalité événementielle 
qui leur impose un coefficient connotatif de nature à orienter leur fonction argumentative. Cette indexicalité est à son tour susceptible de se lexicaliser.

La lecture de l'ouvrage proposé par Sophie Moirand révèle la rigueur théorique et méthodologique de son auteure. Une terminologie appropriée et définie est mise en place. Riche, abondante et nuancée, elle structure l'édifice de manière différentielle et systémique. On aurait souhaité toutefois qu'elle soit plus largement reprise dans l'index de fin d'ouvrage. Il faut reconnaittre que ce dernier aurait pris des proportions qui auraient probablement excédé celles qui lui étaient imparties éditorialement.

La réflexion menée par Sophie Moirand présente une double caractéristique: tournée à la fois vers la modernité (notamment par le renouvellement terminologique qu'elle entreprend et les procédures d'analyse mises en place) et fidèle à l'héritage d'une tradition bakhtinienne d'une part et des apports de l'analyse des discours de vulgarisation de l'autre. C'est ainsi que le concept de dialogisme (cf. plus haut) se voit attribuer une place centrale dans la définition du discours, mais aussi ceux de formation discursive, de domaine de savoir, d'interdiscours, d'intertexte, de genre... La démarche de l'auteur, consciente de cet héritage, n'est pas pour autant «suiviste » et c'est là un des intérêts de la réflexion qu'elle mène. Ainsi la position du sujet comme instance aliénée et clivée par l'histoire n'est pas retenue, contrairement aux prérequis de la conceptologie bakhtinienne. Par ailleurs, une interrogation est menée sur la constitution et l'exploitation des grands corpus, faisant corps ainsi avec les recherches entreprises dans le domaine du traitement automatique. On aurait peutêtre également souhaité qu'internet soit davantage convoqué en tant que vecteur de corpus et que la configuration qu'il impose à la présentation de l'information soit plus fréquemment mise en regard de celle proposée dans la version papier de la presse quotidienne. Ainsi que nous l'avons précisé, cet ouvrage présente une dimension prospective importante; nombre des procédures d'analyses mises en place dans le cadre de la presse quotidienne peuvent être utilisées sur d'autres corpus. Pareillement, l'appareil théorique développé n'est pas limitatif. Il peut être utilisé avec profit sur des corpus appartenant à d'autres champs.

Sommaire de l'ouvrage :

Avant-propos

1. Les choix de l'

- La notion de moment discursif

- Le corpus exploratoire

- L'hétérogénéité des unités discursives

- Les observables de l'analyse

2. La circulation des mots et des formulations

- Des stratégies de désignation

- Les reformulants du prion

- La diffusion du sigle OGM

- La répartition générique des reformulations 
- Des stratégies de médiatisation

- La transmission du sème de la folie

- Les notions émergentes

- Les mots-arguments

- De la monstration à l'allusion

- Du rôle des guillemets et de leur abandon

- L'allusion par les mots et les constructions

- Les mots-événements

- Les objets du discours au fil du texte

3. La diversité et la ronde des dires

- Une situation trilogale

- Bref retour sur la méthode

- La mise en scène des trois pôles

- La voix de la science telle qu'on la montre

- Une parole située

- Des sources qu'on efface

- Un intertexte à plusieurs voix

- Une construction plurilogale

- Les communautés convoquées

- L'inscription des controverses

- Discours masqués ou discours cachés?

- Des lieux d'inscription discrets

- Des discours qu'on évoque ou qu'on imagine

- De la clandestinité des discours transverses

- L'épaisseur dialogique inscrite au fil du texte

4. Mémoires et médias

- Un modèle dialogique de l'explication

- Une interaction plus ou moins représentée

- Différentes formes de dialogisme

- Le surdestinataire du discours médiatique

- Mémoires discursives et mémoires collectives

- L'ancrage discursif de la mémoire

- L'émergence des domaines de mémoire

- La mémoire des mots

- La mémoire des dires

- La construction des mémoires collectives

- L'explication médiatique au fil du texte

En guise d'épilogue

Annexes

Références bibliographiques

Index des notions 